\title{
THÉORIE DE SULLIVAN POUR LA COHOMOLOGIE A COEFFICIENTS LOCAUX
}

\author{
ANTONIO GÓMEZ TATO
}

\begin{abstract}
To every finite dimensional algebraic coefficient system (defined below) $(\Theta, V)$ over the De Rham algebra $\Omega(M)$ of a manifold $M$, Sullivan builds a local system $\rho_{\Theta}: \pi_{1}(M) \rightarrow V$, in the topological sense, such that the two cohomologies $H_{\rho_{\Theta}}^{*}(M ; V)$ and $H_{\Theta}^{*}(\Omega(M) ; V)$ are isomorphic. In this paper, if $\mathbf{K}$ is a simplicial set and $(\boldsymbol{\Theta}, V)$ an algebraic system over the $C^{\infty}$ forms $A_{\infty}(\mathbf{K})$, we prove a similar result. We use it to extend the Hirsch lemma to the case of fibration whose fiber is an Eilenberg-Mac Lane space with certain non nilpotent action of the fundamental group of the basis. We apply this to a model of the hyperbolic torus; different from the nilpotent one, this new model is a better mirror of the topology.
\end{abstract}

\section{INTRODUCTION}

La théorie du modèle minimal de Sullivan [Su] s'est développée autour des notions d'espace nilpotent et d'algèbre minimale nilpotente. Elle a ainsi donné de nombreuses applications dans des domaines variés. Cependant, l'article de Sullivan contenait une notion plus générale qui, à notre connaissance, n'a pas été reprise ultérieurement; il s'agit des algèbres résolubles et des systèmes de coefficients algébriques. Le but de ce travail est de reprendre leur étude et de les relier aux systèmes locaux de coefficients topologiques.

Dans le paragraphe 2, nous donnons les définitions de:

-système algébrique de coefficients, noté $(\Theta, V)$, sur une algèbre différentielle graduée commutative $B$;

-cohomologie de $B$ à valeurs dans le système $(\Theta, V)$, notée $H_{\Theta}^{*}(B ; V)$.

Dans le paragraphe 3 , nous nous intéressons à un cas particulier: celui d'un système algébrique $(\Theta, V)$ de dimension finie, défini sur l'algèbre de De Rham $\Omega(M)$ d'une variété $M$. Dans [Su], Sullivan associe à chaque $(\Theta, V)$ ainsi défini un système de coefficients topologiques, $\rho_{\Theta}: \pi_{1}(M) \rightarrow \operatorname{Aut}_{\mathbf{R}}(V)$. De plus la cohomologie de l'algèbre des formes sur $M$ à valeurs dans $(\Theta, V)$ est isomorphe à la cohomologie de $M$ à valeurs dans le système local topologique $\rho_{\boldsymbol{\theta}}$. Nous rappelons cette construction et donnons un cas particulier de retour de la topologie vers l'algèbre, utilisé dans le dernier paragraphe.

Dans le paragraphe 4, nous obtenons des résultats analogues dans le cadre des ensembles simpliciaux (Théorème 4.7). Nous utilisons pour cela la notion de

Received by the editors August 2, 1988 and, in revised form, December 27, 1989.

1980 Mathematics Subject Classification (1985 Revision). Primary 55N25, 55P62, 57N65. 
système local de coefficients sur un ensemble simplicial telle qu'elle est définie dans [Ha].

Le paragraphe 5 est consacré à une généralisation du lemme de Hirsch. Rappelons brièvement de quoi il s'agit: soit $F \rightarrow E \rightarrow X$ une fibration, où la fibré $F$ est un espace d'Eilenberg-Mac Lane de cohomologie réelle $\Lambda V$, transgressive. Le lemme de Hirsch classique implique l'existence d'une différentielle $D$ et d'une flèche $\Gamma$ induisant un isomorphisme en cohomologie réelle:

$$
\Gamma:\left(A_{\infty}(X) \otimes \Lambda V, D\right) \rightarrow A_{\infty}(E) .
$$

Ici, si l'action de $\pi_{1}(X)$ sur $V$ provient d'un système algébrique de coefficients, on démontre l'existence de $\Gamma$ et $D$ comme ci-dessus et telles que $\Gamma$ induise un isomorphisme pour toutes les cohomologies à coefficients finis provenant de $A_{\infty}(X)$ (Théorème 5.1).

Au paragraphe 6, nous appliquons cela au tore hyperbolique, espace total d'une fibration principale de fibre un tore $S^{1} \times S^{1}$ et de base $S^{1}$ [GS]. Le type d'homotopie rationnelle obtenu en réalisant un modèle minimal nilpotent de son algèbre des formes simpliciales coïncide avec celui du produit $S^{1} \times S^{2}$. Nous voyons apparaître ici un exemple où la théorie nilpotente ne rend pas compte de la topologie de l'espace. Par contre, la construction de son modèle suivant les méthodes du paragraphe précédent fournit deux générateurs de degré 1 correspondant à la fibre.

Cet article reprend des résultats de ma thèse réalisée sous la direction de Daniel Tanré; je le remercie pour son aide qui m'a permis de surmonter plusieurs difficultés. Ce travail a profité de l'ambiance stimulante du Laboratoire de Géométrie et Topologie de l'Université des Sciences et Techniques de LilleFlandre-Artois et du soutien de Xosé Masa Professeur à l'Université de Santiago. Je remercie également le referee pour ses remarques qui ont contribué à améliorer le texte.

\section{Systèmes ALGÉBRIQUeS DE COEFFICIENTS}

Soit $k$ un corps de caractéristique zéro. Notons $k$-ADG la catégorie des $k$ algèbres différentielles graduées associatives et $k-\mathrm{ADG}_{\mathrm{c}}$ la sous-catégorie pleine formée des algèbres commutatives (au sens gradué); rappelons que $B=\bigoplus_{p} B^{p}$ est un objet de $k-\mathrm{ADG}_{\mathrm{c}}$ si la multiplication $B^{p} \otimes B^{q} \rightarrow B^{p+q}$, qui à $x \otimes y$ associe $x y$, vérifie $x y=(-1)^{p q} y x$. Nous utiliserons l'expression adg (resp. adgc) pour désigner un objet de $k$-ADG (resp. $k-A^{-} G_{c}$ ). Nous suivrons les règles usuelles de commutation d'objets gradués; si $x$ est un élément homogène d'un objet gradué, nous noterons son degré par $|x|$.

Soit $B=(B, d)$ une adgc; pour simplifier les notations, $B$ désignera aussi l'algèbre sous-jacente à l'adgc $B$. L'algèbre $B$ étant commutative, tout $B$ module gradué à droite (resp. à gauche) $Y$ peut être vu comme un $B$-module à gauche (resp. à droite) par: $b y=(-1)^{|b|}|y| y b$; dans la suite, nous dirons que $Y$ est un $B$-module gradué. Un $B$-module différentiel gradué $Y$ est un $B$-module gradué muni d'une différentielle de $B$-module, c'est-à-dire, d'une application de $k$-espaces gradués $d: Y \rightarrow Y$ de degré +1 telle que $d^{2}=0$ et $d(y b)=(d y) b+(-1)^{|y|} y d b$. Une $B$-adg est la donnée d'un morphisme $B \rightarrow A$ de $k$-ADG; elle définit une structure de $B$-module différentiel gradué sur $A$. Dans le cas particulier où $A \in k-\mathrm{ADG}_{\mathrm{c}}$, nous parlerons de $B$-adgc. 
Si $W$ est un $k$-espace vectoriel concentré en degré zéro et $(Y, d)$ un $B$ module différentiel gradué, le produit tensoriel, comme $k$-modules, $Y \otimes W$ a une structure de $B$-module différentiel gradué donnée par $(y \otimes w) b=(y b \otimes w)$, $d(y \otimes w)=d y \otimes w$. Dans le cas particulier où $Y=B$ et $W$ est l'espace des endomorphismes d'un $k$-espace vectoriel $V, W=\operatorname{End}(V)$, nous obtenons une structure de $B$-adg sur $B \otimes \operatorname{End}(V)$ avec la multiplication

$$
(b \otimes f) \circ(c \otimes g)=b c \otimes f \circ g .
$$

Définition 2.1. Un système algébrique de coefficients sur une adgc $B$ est une paire $(\Theta, V)$ constituée d'un $k$-espace vectoriel $V$ (concentré en degré zéro) et d'un élément $\Theta$, de degré 1 , de la $B$-adg $B \otimes \operatorname{End}(V)$ vérifiant:

$$
d \boldsymbol{\Theta}+\boldsymbol{\Theta} \circ \boldsymbol{\Theta}=0 .
$$

Nous dirons aussi que $(\Theta, V)$ est un système sur $B$; la dimension du système est celle de l'espace vectoriel $V$.

Un système $(\Theta, V)$ sur $B$, peut être vu comme une application $\Theta: V \rightarrow$ $B^{1} \otimes V . \operatorname{Si} \operatorname{dim}(V)=n<\infty$ et si $\left\{v_{1}, \ldots, v_{n}\right\}$ est une base pour $V$, notons $\theta=\left(\theta_{i j}\right)$ la matrice d'éléments de $B^{1}$ telle que $\Theta\left(v_{i}\right)=\sum \theta_{i j} \otimes v_{j}$. La matrice d'éléments de $B^{2}$ associée à $\Theta \circ \Theta$ est la matrice produit $-\theta \theta$. L'équation (1) devient alors $d \theta=\theta \theta$.

Si $(Y, d)$ est un $B$-module différentiel gradué et $V$ un $k$-espace vectoriel (concentré en degré zéro), nous définissons une action de $B \otimes V$ sur $Y$ par $y(b \otimes v)=y b \otimes v$. La donnée d'un système $(\Theta, V)$ sur $B$ fournit une dérivation $d_{\Theta}$ sur le $B$-module $Y \otimes V$ par $d_{\Theta}(y \otimes v)=d y \otimes v+(-1)^{|y|} y \Theta(v)$. L'équation (1) entraîne $d_{\Theta}^{2}=0$ et $d_{\Theta}$ est donc une différentielle de $B$-module.

Définition 2.2. L'homologie du complexe $\left(Y \otimes V, d_{\Theta}\right)$ est appelée cohomologie de $(Y, d)$ à valeurs dans le système $(\Theta, V)$; on la note $H_{\Theta}^{*}(Y ; V)$.

Remarque 2.3. Constatons pour terminer que la donnée d'un système sur l'adgc $B$ équivaut à celle d'une différentielle de $B$-module sur $B \otimes V$.

\section{RAPPORT ENTRE LES SYSTEMES ALGÉBRIQUES ET TOPOLOGIQUES DE COEFFICIENTS: CAS DES VARIÉTÉS DIFFÉRENTIABLES}

Illustrons les définitions précédentes en montrant comment un système algébrique donne naissance à un système local de coefficients au sens habituel.

Soit $M$ une variété réelle $C^{\infty}$. Notons $\Omega(M)$ l'algèbre des formes $C^{\infty}$ sur $M$. Dans la suite, $k$ est le corps $\mathbf{R}$ des réels.

Définition 3.1. Un système algébrique de coefficients sur $M$ est un système $(\Theta, V)$ sur l'adgc $\Omega(M)$.

Soit $\eta$ un fibré vectoriel sur $M$, de fibre type l'espace vectoriel réel $V$; on note $\Omega(M ; \eta)$ le $\Omega(M)$-module des formes différentielles sur $M$ à valeurs dans $\eta$. Si $\eta$ est un fibré trivialisable sur $M$, la trivialisation donne un isomorphisme $\Omega(M ; \eta) \cong \Omega(M) \otimes V$ de $\Omega(M)$-modules.

Rappelons maintenant que la donnée d'un système algébrique $(\Theta, V)$ sur $M$ équivaut à celle d'une différentielle de $\Omega(M)$-module $d_{\Theta}$ sur $\Omega(M) \otimes V$. Géométriquement, celle-ci correspond à une connexion linéaire plate sur le fibré trivial $\eta: M \times V \rightarrow M$ [GHV, 7.11]. En utilisant le transport par parallélisme, 
une telle connexion peut aussi être vue comme une représentation $\rho_{\Theta}: \pi_{1}(M) \rightarrow$ $\operatorname{Aut}_{\mathbf{R}}(V)$ telle que le fibré vectoriel plat induit sur $M$ soit trivialisé.

Définition 3.2. Un système topologique de coefficients locaux sur $M,(V, \rho)$, est une représentation $\rho: \pi_{1}(M) \rightarrow \operatorname{Aut}_{\mathbf{R}}(V)$.

Notons $\xi$ le fibré vectoriel plat induit par $\rho$. Par platitude de $\xi$, la dérivée covariante le long de la connexion, $\nabla_{\rho}$, est une différentielle pour $\Omega(M ; \xi)$. Remarquons, avec ce qui précède, que l'existence d'un système algébrique $(\Theta, V)$ sur $\Omega(M)$ tel que $(V, \rho)=\left(V, \rho_{\Theta}\right)$ équivaut à la trivialisabilité de $\xi$. Nous posons donc:

Définition 3.3. Un système topologique $(V, \rho)$ provient d'un système algébrique si le fibré vectoriel plat associé à $\rho$ trivialise. Dans ce cas, on dit que $\rho$ est une action algébrique et on note $\rho=\rho_{\Theta}$, où $(\Theta, V)$ est un système algébrique donné par la trivialisation.

Définition 3.4. L'homologie du complexe $\left(\Omega(M ; \xi), \nabla_{\rho}\right)$ est appelée cohomologie de $M$ à valeurs dans le système $(V, \rho)$; on la note $H_{\rho}^{*}(M ; V)$.

Théorème 3.5 (Sullivan). Soit $(V, \rho)$ un système topologique sur $M$ provenant d'un système algébrique $(\Theta, V)$ sur $\Omega(M)$. Alors, on a un isomorphisme:

$$
H_{\rho_{\boldsymbol{\theta}}}^{*}(M ; V) \cong H_{\Theta}^{*}(\Omega(M) ; V) .
$$

Notons $\xi$ le fibré vectoriel plat (trivialisé) induit par $\rho$. Avec le rappel précédent, la démonstration [Su] consiste à remarquer l'existence d'un isomorphisme au niveau des complexes:

$$
\left(\Omega(M ; \xi), \nabla_{\rho_{\boldsymbol{\theta}}}\right) \cong\left(\Omega(M) \otimes V, d_{\boldsymbol{\theta}}\right) .
$$

Dans le cas $\pi_{1}(M)=\mathbf{Z}$, on a un retour de la topologie vers l'algèbre; nous l'utiliserons au paragraphe 6 .

Proposition 3.6. Soit $M$ une variété $C^{\infty}$ de groupe fondamental $\mathbf{Z}$, munie d'un système topologique de coefficients $(V, \rho)$, de dimension finie. Choisissons un générateur, noté 1 , de $\pi_{1}(M)$. Si $\rho(1)$ est un isomorphisme à déterminant positif, alors le système topologique provient d'un système algébrique $(\Theta, V)$.

Preuve. Il suffit de remarquer que, sous l'hypothèse $\rho(1)$ à déterminant positif, le fibré vectoriel associé trivialise.

Corollaire 3.7. Avec les hypothèses de la proposition, si $\rho(1)$ est une application exponentielle, le système algébrique $\Theta$ est donné par

$$
\Theta=\alpha \otimes \log (\rho(1))
$$

où $\alpha$ est une 1-forme fermée explicitée dans la démonstration.

Preuve. Soit $\gamma$ un lacet différentiable plongé représentant le générateur de $\pi_{1}(M)=\mathbf{Z}$. Choisissons $\alpha \in \Omega^{1}(M)$ telle que $\alpha_{/ \gamma}$ soit la forme volume standard $d t$. Regardons $d_{\Theta}$ comme une connexion sur $M \times V$. Alors le déplacement par parallélisme le long de $\gamma$ est exactement $\exp (\log \rho(1))=\rho(1)$, et par conséquent $\rho_{\boldsymbol{\Theta}}=\rho$.

Remarque 3.8. Ce problème du passage de la topologie à l'algèbre est résolu dans [DS] pour $k=\mathbf{C}$; les auteurs y montrent que tout fibré vectoriel complexe à groupe structural discret, de base un polyèdre compact $B$, devient trivial après passage à un revêtement fini de $B$. 


\section{RAPPORT ENTRE LES SYSTĖMES ALGÉBRIQUES \\ ET TOPOLOGIQUES DE COEFFICIENTS: \\ CAS DES ESPACES SIMPLICIAUX}

Rappelons d'abord les définitions suivantes de [Ha]; $C$ dénote soit la catégorie des $k$-espaces vectoriels gradués différentiels, soit $k-\mathrm{ADG}_{\mathrm{c}}$.

Fixons un ensemble simplicial $\mathbf{K}$; si $\sigma \in \mathbf{K}_{n}$, on note sa dimension par $|\sigma|=n$.

Définition 4.1. Un système local $F$ sur $\mathbf{K}$ à valeurs dans $C$ est la donnée:

-d'une famille d'objets $F_{\sigma}$ de $C, \sigma \in \mathbf{K}$,

-et d'une famille de morphismes (appelés opérateurs face et dégénérescence)

$$
\partial_{i}: F_{\sigma} \rightarrow F_{\partial_{i} \sigma} \text { et } s_{j}: F_{\sigma} \rightarrow F_{s_{j} \sigma}
$$

induisant des isomorphismes en cohomologie et vérifiant les habituelles formules de commutation:

$$
\begin{aligned}
\partial_{i} \partial_{j} & =\partial_{j-1} \partial_{i}, \quad i<j, \\
s_{i} s_{j} & =s_{j+1} s_{i}, \quad i \leq j,
\end{aligned}
$$

et

$$
\partial_{i} s_{j}= \begin{cases}s_{j-1} \partial_{i}, & i<j, \\ \mathrm{id}, & i=j, j+1, \\ s_{j} \partial_{i-1}, & i>j+1 .\end{cases}
$$

Remarque 4.2. Cette définition est celle de système local de coefficients différentiels de [Ha].

Si $\phi: \mathbf{L} \rightarrow \mathbf{K}$ est une application simpliciale, on obtient un système local sur $\mathbf{L}$ avec $\left(\phi^{*} F\right)_{\sigma}=F_{\phi(\sigma)} ; \partial_{i}=\partial_{i} ; s_{j}=s_{j}$. Il est noté $\phi^{*} F$ et appelé image réciproque de $F$ par $\phi$.

Définition 4.3. L'espace des sections globales de $F$, noté $F(\mathbf{K})$, est l'ensemble des éléments $\Phi$, associant à chaque $\sigma \in \mathbf{K}$ un élément $\Phi_{\sigma} \in F_{\sigma}$ tel que: $\boldsymbol{\Phi}_{\partial_{i} \sigma}=\partial_{i} \boldsymbol{\Phi}_{\sigma}$ et $\boldsymbol{\Phi}_{s_{j} \sigma}=s_{j} \boldsymbol{\Phi}_{\sigma}$.

Remarquons que $F(\mathbf{K})$ est naturellement un objet de $C$.

Définition 4.4. La cohomologie de $F(\mathbf{K})$ est appelée cohomologie de $\mathbf{K}$ à valeurs dans le système local $F$ et notée $H^{*}(F(\mathbf{K}))$.

On note $\Delta^{n}$ le $n$-simplexe standard (regardé comme un ensemble simplicial). Soit $\left[\Delta^{n}\right]$ le seul $n$-simplexe non dégénéré de $\Delta^{n}$. Si $\mathbf{K}$ est un ensemble simplicial, chaque $\sigma \in \mathbf{K}_{n}$ détermine une seule application simpliciale $\sigma: \Delta^{n} \rightarrow \mathbf{K}$ telle que $\sigma\left(\left[\Delta^{n}\right]\right)=\sigma[\mathrm{Ha}, 12.12]$.

Définition 4.5. Le système local $F$ est extensible si, pour tout $\sigma \in \mathbf{K}_{n}$, la restriction

$$
\boldsymbol{\sigma}^{*} F\left(\Delta^{n}\right) \rightarrow \sigma^{*} F\left(\boldsymbol{\partial} \Delta^{n}\right)
$$

est surjective.

Une forme $C^{\infty}$ sur le $n$-simplexe géométrique standard $\Delta^{n} \subset \mathbf{R}^{n+1}$ est la restriction à $\Delta^{n}$ d'une forme $C^{\infty}$ définie sur un voisinage; notons $e_{0}, e_{1}, \ldots, e_{n}$ les sommets de $\Delta^{n}$. L'algèbre des formes $C^{\infty}$ sur $\Delta^{n}$ est notée $A_{\infty}(n)$. Soient $\partial_{i}: \Delta^{n-1} \rightarrow \Delta^{n}, s_{j}: \Delta^{n+1} \rightarrow \Delta^{n}$ les opérateurs $i$-face, $j$-dégénérescence habituels, 
alors le système local des formes différentielles $A_{\infty}$ sur $\mathbf{K}$ est défini par [Ha, Chapitre 13]:

$$
\left(A_{\infty}\right)_{\sigma}=A_{\infty}(|\sigma|), \quad \partial_{i}=A_{\infty}\left(\partial_{i}\right), \quad s_{j}=A_{\infty}\left(s_{j}\right) .
$$

A partir de là, on associe à tout système local $F$ sur $\mathbf{K}$ un nouveau système, noté $A_{\infty} \otimes F$, défini par:

$$
\left(A_{\infty} \otimes F\right)_{\sigma}=A_{\infty}(|\sigma|) \otimes F_{\sigma}, \quad \partial_{i} \otimes \partial_{i}, \quad s_{j} \otimes s_{j} .
$$

L'espace des sections globales de $A_{\infty} \otimes F$, noté $A_{\infty}(\mathbf{K} ; F)$ est appelé espace des formes différentielles sur $\mathbf{K}$ à valeurs dans le système local $F$. Son homologie est notée $H^{*}\left(A_{\infty}(\mathbf{K} ; F)\right)$.

Considérons maintenant un système algébrique $(\Theta, V)$ sur $A_{\infty}(\mathbf{K})$ de dimension $l$. Par restriction, on obtient pour chaque $\sigma \in \mathbf{K}_{n}$, un système $\left(\Theta_{\sigma}, V\right)$ sur $A_{\infty}(|\sigma|)$, donc une connexion plate sur le fibré trivial $\Delta^{n} \times V \rightarrow \Delta^{n}$. Notons la aussi $\boldsymbol{\Theta}_{\sigma}$; elle est difféomorphe à la connexion standard car $\Delta^{n}$ est contractile. Comme conséquence immédiate de la dimension finie de $V$ on a:

Proposition 4.6. Le complexe $\left(A_{\infty}(\mathbf{K}) \otimes V, d_{\mathbf{\Theta}}\right)$ est l'espace des sections globales du système local $A_{\infty} \otimes_{\tau} V$, défini par:

$$
\left(A_{\infty} \otimes_{\tau} V\right)_{\sigma}=\left(A_{\infty}(|\sigma|) \otimes V, d_{\boldsymbol{\Theta}_{\sigma}}\right), \quad \partial_{i} \otimes \mathrm{id}, \quad s_{j} \otimes \mathrm{id} .
$$

Théorème 4.7. Soit $\mathbf{K}$ un ensemble simplicial et soit $(\Theta, V)$ un système algébrique de coefficients sur $A_{\infty}(\mathbf{K})$. Alors, il existe un système local $V_{\mathbf{\Theta}}$ sur $\mathbf{K}$ et un isomorphisme naturel:

$$
H_{\boldsymbol{\Theta}}^{*}\left(A_{\infty}(\mathbf{K}) ; V\right) \cong H^{*}\left(A_{\infty}\left(\mathbf{K} ; V_{\mathbf{\Theta}}\right)\right) .
$$

Preuve. Construisons d'abord le système $V_{\Theta}$. Pour cela, soit $\Delta^{n} \times V \rightarrow \Delta^{n}$ le fibré trivial muni de la connexion $\boldsymbol{\Theta}_{\sigma}, \sigma \in \mathbf{K}_{n}$; le déplacement le long du chemin $t e_{1}+(1-t) e_{0}$ induit un isomorphisme $\rho(\sigma)$ entre les fibres $\left\{e_{0}\right\} \times V$ et $\left\{e_{1}\right\} \times V$. Par abus de notation, $\rho(\sigma)$ désigne aussi l'automorphisme induit sur $V$.

Définissons le système local $V_{\boldsymbol{\Theta}}$, sur $\mathbf{K}$ par:

$V_{\sigma}=V, \sigma \in \mathbf{K}_{n}$,

$\partial_{i}: V_{\sigma} \rightarrow V_{\partial_{i} \sigma}$ est l'identité si $i \neq 0$ et $\partial_{0}=\rho(\sigma)$,

$s_{j}=$ id pour tout $j$.

Notons que $V_{\Theta}$ est un système local car le déplacement par parallélisme entre deux points de $\Delta^{n}$ ne dépend pas du chemin choisi.

Soit $A_{\infty} \otimes_{\tau} V$ le système local défini en 4.6. Si on montre:

$$
\left(A_{\infty}\left(\mathbf{K} ; V_{\mathbf{\Theta}}\right), d\right) \cong\left(A_{\infty} \otimes_{\tau} V\right)(\mathbf{K})
$$

avec la Proposition 4.6 on aura fini.

Pour cela on considère pour chaque $n$-simplexe $\sigma$ le fibré vectoriel trivial $\Delta^{n} \times V \rightarrow \Delta^{n}$ muni de la connexion plate $\boldsymbol{\Theta}_{\sigma}$. Chaque vecteur $v \in\left\{e_{\sigma}\right\} \times V$ s'étend en une section parallèle $\gamma_{\sigma}(v)$ du fibré; on définit un isomorphisme $A_{\infty}(n) \otimes V \stackrel{\gamma_{\sigma}}{\rightarrow} A_{\infty}(n) \otimes V$ par $\gamma_{\sigma}(w \otimes v)=w \wedge \gamma_{\sigma}(v)$. L'ensemble $\left\{\gamma_{\sigma}\right\}_{\sigma \in \mathbf{K}}$ définit l'isomorphisme cherché entre $A_{\infty} \otimes V_{\Theta}$ et $A_{\infty} \otimes_{\tau} V$.

Remarque 4.8. Soit $X$ un espace topologique 0 -connexe; on note $A_{\infty}(X)$ l'algèbre des sections globales du système local $A_{\infty}$ sur l'ensemble simplicial $\operatorname{Sing}(X)$. 
$\mathrm{Si}(\Theta, V)$ est un système algébrique de coefficients sur $A_{\infty}(X)$, on construit le système local $V_{\boldsymbol{\theta}}$; soit $\rho_{\boldsymbol{\Theta}}: \pi_{1}(X) \rightarrow \operatorname{Aut}_{\mathbf{R}}(V)$ le système topologique défini par $V_{\Theta}$ [Ha, Chapitre 16]. Notons $H_{\rho_{\theta}}^{*}(X ; V)$ la cohomologie à coefficients locaux associée à $\rho_{\Theta}$; la forme particulière de $V_{\Theta}$ entraîne:

$$
H^{*}\left(C^{*}\left(\operatorname{Sing}(X) ; V_{\boldsymbol{\theta}}\right)\right) \cong H_{\rho_{\boldsymbol{\theta}}}^{*}(X ; V) .
$$

D'autre part, le théorème de De Rham fournit un isomorphisme

$$
H^{*}\left(C^{*}\left(\operatorname{Sing}(X) ; V_{\boldsymbol{\Theta}}\right)\right) \cong H^{*}\left(A_{\infty}\left(\operatorname{Sing}(X) ; V_{\boldsymbol{\Theta}}\right)\right),
$$

d'où, d'après le Théorème 4.7 et par composition des isomorphismes précédents:

$$
H_{\rho_{\boldsymbol{\theta}}}^{*}(X ; V) \cong H_{\boldsymbol{\Theta}}^{*}\left(A_{\infty}(X) ; V\right) .
$$

\section{LEMME de HiRsch}

Dans ce paragraphe, soit $\pi$ un groupe abélien tel que $V=\pi \otimes_{\mathbf{Z}} \mathbf{R}$ soit de dimension $n$. L'espace d'Eilenberg-Mac Lane ayant $\pi$ comme groupe d'homotopie de degré $m$ est noté $K(\pi, m)$. On va établir ici une généralisation du lemme de Hirsch [Hi] au cas d'une fibration de fibre $K(\pi, m)$, munie d'un type particulier d'action (non nécessairement nilpotente) du groupe fondamental de la base sur $\pi$.

Fixons un espace 0-connexe $X$ et $p: E \rightarrow X$ une fibration à fibre $F=$ $K(\pi, m), m \geq 1$. Le morphisme de groupes $\pi \stackrel{j}{\rightarrow} \pi \otimes_{\mathbf{Z}} \mathbf{R}$ fournit une inclusion $\operatorname{Aut}_{\mathbf{Z}}(\pi) \subset \operatorname{Aut}_{\mathbf{R}}(V)$. Ainsi, l'action $\rho: \pi_{1}(X) \rightarrow \operatorname{Aut}_{\mathbf{Z}}(\pi)$ s'étend en une action, notée aussi $\rho, \rho: \pi_{1}(X) \rightarrow \operatorname{Aut}_{\mathbf{R}}(V)$. Nous supposons qu'elle provient d'un système algébrique $(\Theta, V)$ sur $A_{\infty}(X)$. Notons $(\pi, \rho),(V, \rho)$ les systèmes locaux topologiques définis par $\rho$.

Le Théorème des Coefficients Universels donne un isomorphisme

$$
\Phi_{1}: H^{m}(F ; \pi) \rightarrow \operatorname{Hom}_{\mathbf{Z}}\left(H_{m}(F), \pi\right)
$$

et le Théorème de Hurewicz un isomorphisme

$$
\Phi_{2}: \operatorname{Hom}_{\mathbf{Z}}\left(H_{m}(F), \pi\right) \rightarrow \operatorname{Hom}_{\mathbf{Z}}(\pi, \pi) ;
$$

notons $\Phi=\Phi_{2} \circ \Phi_{1}$. Soient $\Delta F=\Phi^{-1}$ (id) la classe fondamentale de $F$ et $c(p) \in H_{\rho}^{m+1}(X, * ; \pi)$ la classe caractéristique de la fibration $p$.

Considérons le diagramme commutatif suivant:

$$
\begin{array}{llll}
H^{m}(F ; \pi) \stackrel{\partial}{\longrightarrow} H_{p^{*} \rho}^{m+1}(E, F ; \pi) & \rightarrow & H_{p^{*} \rho}^{m+1}(E ; \pi) \\
& H_{\rho}^{m+1}(X, * ; \pi) & \rightarrow & H_{\rho}^{m+1}(X ; \pi)
\end{array}
$$

où la ligne supérieure est extraite de la suite exacte longue de cohomologie de la paire $(E, F)$.

D'après [Ba, 5.2.9], la classe $c(p)$ est la transgression de $\Delta F$; c'est-à-dire: $\tilde{p}^{*} c(p)=\partial \Delta F$.

Si on prend la cohomologie à valeurs dans le système local $(V, \rho)$ on obtient un diagramme commutatif:

$$
\begin{aligned}
& H^{m}(F ; V) \stackrel{\partial}{\longrightarrow} H_{p^{*} \rho}^{m+1}(E, F ; V) \rightarrow H_{p^{*} \rho}^{m+1}(E ; V) \\
& \begin{array}{c}
\uparrow \tilde{p}^{*} \\
H_{\rho}^{m+1}(X, * ; V)
\end{array} \rightarrow H_{\rho}^{m+1}(X ; V)
\end{aligned}
$$


relié au diagramme précédent par des morphismes induits par l'inclusion $\pi \stackrel{j}{\rightarrow}$ $\pi \otimes_{\mathbf{Z}} \mathbf{R}$. On note $\overline{c(p)}, \overline{\Delta F}$ les images de $c(p), \Delta F$ dans $H_{\rho}^{m+1}(X, * ; V)$ et $H^{m}(F, V)$ respectivement. Par naturalité elles vérifient toujours la relation $\partial \overline{\Delta F}=\tilde{p}^{*} \overline{c(p)}$.

D'après la Remarque 4.8 et le fait que $(V, \rho)$ provienne d'un système $(\Theta, V)$, le diagramme précédent s'écrit aussi:

$$
\begin{array}{rlll}
H^{m}\left(A_{\infty}(F) ; V\right) \stackrel{\partial}{\longrightarrow} H_{\boldsymbol{\Theta}}^{m+1}\left(A_{\infty}(E, F) ; V\right) & \rightarrow & H_{\boldsymbol{\Theta}}^{m+1}\left(A_{\infty}(E) ; V\right) \\
& H_{\boldsymbol{\Theta}}^{m+1}\left(A_{\infty}(X, *) ; V\right) & \rightarrow & H_{\boldsymbol{\Theta}}^{m+1}\left(A_{\infty}(X) ; V\right) .
\end{array}
$$

La ligne supérieure s'obtient à partir de la suite exacte courte de $A_{\infty}(X)$ modules:

$$
0 \rightarrow\left(A_{\infty}(E, F) \otimes V, d_{\boldsymbol{\theta}}\right) \stackrel{\alpha}{\longrightarrow}\left(A_{\infty}(E) \otimes V, d_{\boldsymbol{\theta}}\right) \stackrel{i}{\longrightarrow}\left(A_{\infty}(F) \otimes V, d_{\boldsymbol{\theta}}\right) \rightarrow 0 .
$$

Soient $x \in A_{\infty}^{m+1}(X, *) \otimes V, y \in A^{m}(F) \otimes V$ des $d_{\Theta}$-cocycles tels que $[x]=\overline{c(p)},[y]=\overline{\Delta F}$. Comme $\partial[y]=\tilde{p}^{*}[x]$, on peut choisir $\mu \in A_{\infty}(E) \otimes V$ tel que $i(\mu)=y$ et $d_{\Theta} \mu=\alpha(\tilde{p}(x))$. Soit $V^{*}$ l'espace dual de $V$; on choisit une base $\left\{v_{1}, \ldots, v_{n}\right\}$ de $V$ et on note $\left\{v_{1}^{*}, \ldots, v_{n}^{*}\right\}$ la base duale; on peut écrire:

$$
\mu=\sum_{i=1}^{n} \mu_{i} \otimes v_{i}, \quad \mu_{i} \in A_{\infty}^{m}(E), \quad \text { et } \quad x=\sum_{i=1}^{n} x_{i} \otimes v_{i}, \quad x_{i} \in A_{\infty}^{m+1}(X, *) .
$$

Notons $\left(\Theta^{t}, V^{*}\right)$ le système défini par $\Theta^{t}\left(v_{i}^{*}\right)=-\sum \theta_{j i} \otimes v_{j}^{*}$ où $\left(\theta_{i j}\right)$ est la matrice associée à $(\Theta, V)$ par rapport à la base choisie. On note $A_{\infty}(X) \otimes_{\tau}$ $\Lambda\left(V^{*}\right)$ l'extension de $A_{\infty}(X)$ par l'algèbre libre engendrée par $V^{*}$ en degré $m$, munie de la différentielle tordue définie sur les générateurs par

$$
d(a \otimes 1)=d a \otimes 1, \quad d\left(1 \otimes v_{i}^{*}\right)=\Theta^{t}\left(v_{i}^{*}\right)+x_{i} .
$$

(On a gardé la notation $x_{i}$ pour l'image de $x_{i} \in A_{\infty}^{m+1}(X, *)$ dans $A_{\infty}^{m+1}(X)$.)

Soit $\Gamma: A_{\infty}(X) \otimes_{\tau} \Lambda\left(V^{*}\right) \rightarrow A_{\infty}(E)$ le morphisme d'algèbres différentielles défini sur les générateurs par

$$
\Gamma(a \otimes 1)=p^{*}(a) \quad \text { et } \quad \Gamma\left(1 \otimes v_{i}^{*}\right)=\mu_{i} .
$$

On peut maintenant démontrer la généralisation annoncée du lemme de Hirsch:

Théorème 5.1. $\Gamma$ induit un isomorphisme pour toute cohomologie à coefficients dans n'importe quel système algébrique de dimension finie $(\widetilde{\Theta}, W)$ sur $A_{\infty}(X)$. Preuve. Fixons tout d'abord un système $(\widetilde{\Theta}, W)$ sur $A_{\infty}(X)$. On va utiliser le théorème suivant:

Théorème [Ha, 12.27]. Soit $\phi: N_{1} \rightarrow N_{2}$ un morphisme de systèmes locaux extensibles sur un ensemble simplicial $\mathbf{K}$ tel que pour chaque $\sigma, \phi_{\sigma}^{*}: H^{*}\left(\left(N_{1}\right)_{\sigma}\right) \rightarrow$ $H^{*}\left(\left(N_{2}\right)_{\sigma}\right)$ soit un isomorphisme. Alors il existe un morphisme $\phi: N_{1}(\mathbf{K}) \rightarrow$ $N_{2}(\mathbf{K})$, donné par $(\phi \Phi)_{\sigma}=\phi_{\sigma}\left(\Phi_{\sigma}\right)$, et tel que

$$
\phi^{*}: H^{*}\left(N_{1}(\mathbf{K})\right) \rightarrow H^{*}\left(N_{2}(\mathbf{K})\right)
$$

soit un isomorphisme. 
Construisons donc deux systèmes locaux extensibles $R$ et $\widetilde{G}$ sur $\operatorname{Sing}(X)$ tels que $\Gamma$ induise un morphisme entre eux et que l'on ait

$$
\begin{gathered}
R(X) \cong\left(\left(A_{\infty}(X) \otimes_{\tau} \Lambda\left(V^{*}\right)\right) \otimes W, d_{\widetilde{\boldsymbol{\Theta}}}\right), \\
\widetilde{G}(X) \cong\left(A_{\infty}(E) \otimes W, d_{\widetilde{\boldsymbol{\Theta}}}\right) .
\end{gathered}
$$

Le système local $R$ sur $\operatorname{Sing}(X)$ est défini par

$$
R_{\sigma}=\left(\left[A_{\infty}(|\sigma|) \otimes_{\tau_{\sigma}} \Lambda\left(V^{*}\right)\right] \otimes W, d_{\widetilde{\boldsymbol{\Theta}}_{\sigma}}\right), \quad \partial_{i} \otimes \mathrm{id} \otimes \mathrm{id}, \quad s_{j} \otimes \mathrm{id} \otimes \mathrm{id},
$$

où $\partial_{i}: A_{\infty}(|\sigma|) \rightarrow A_{\infty}\left(\left|\partial_{i} \sigma\right|\right)$ et $s_{j}: A_{\infty}(|\sigma|) \rightarrow A_{\infty}\left(\left|s_{j} \sigma\right|\right)$ sont les opérateurs face et dégénérescence du système $A_{\infty}$.

Le système $R$ est extensible car $A_{\infty}$ l'est. Vérifions que $R$ est un système local.

Pour chaque $\sigma$, il existe une suite spectrale [Gr], obtenue en filtrant par le degré en $A_{\infty}(|\sigma|)$. Elle converge vers la cohomologie de $R_{\sigma}$ et son terme $E_{2}$ s'écrit:

$$
E_{2}^{p, q}=H_{\bar{\Theta}_{\sigma}}^{p}\left(A_{\infty}(|\sigma|) ;\left(\Lambda\left(V^{*}\right) \otimes W\right)^{q}\right),
$$

$\left(\overline{\boldsymbol{\Theta}}_{\sigma},\left(\Lambda\left(V^{*}\right) \otimes W\right)^{q}\right)$ étant un système sur $A_{\infty}(|\sigma|)$ construit à partir de $\left(\boldsymbol{\Theta}^{t}, V^{*}\right)$ et $(\widetilde{\Theta}, W)$. De la contractilité de l'espace $\Delta^{|\sigma|}$ et du Théorème 4.7, on déduit:

$$
E_{2}^{p, q}= \begin{cases}\left(\Lambda\left(V^{*}\right) \otimes W\right)^{q}, & p=0, \\ 0, & p>0 .\end{cases}
$$

On vérifie que $\partial_{i} \otimes \mathrm{id} \otimes \mathrm{id}$ et $s_{j} \otimes \mathrm{id} \otimes \mathrm{id}$ respectent les filtrations; le calcul de la suite spectrale montre donc que $R$ est un système local. Les espaces $V, W$ étant de dimension finie, $R(X)$ admet une base de sections globales, comme $A_{\infty}(X)$-module, et on obtient

$$
R(X) \cong\left(\left(A_{\infty}(X) \otimes_{\tau} \Lambda\left(V^{*}\right)\right) \otimes W, d_{\widetilde{\boldsymbol{\Theta}}}\right) .
$$

Passons à la construction de $\widetilde{G}$; pour cela, rappelons la construction d'un système local extensible $G$ sur $\operatorname{Sing}(X)$, associé à la fibration $p: E \rightarrow X[\mathrm{Ha}$, 19.16].

Pour chaque $\sigma: \Delta^{n} \rightarrow X$, soit $p_{\sigma}: E_{\sigma} \rightarrow \Delta^{n}$ la fibration image réciproque de $p$. Notons $\mathbf{E}_{\sigma} \subset \operatorname{Sing}\left(E_{\sigma}\right)$ le sous ensemble simplicial de $\operatorname{Sing}\left(E_{\sigma}\right)$ défini par: $\tau \in \mathbf{E}_{\sigma} \Leftrightarrow p_{\sigma} \circ \tau \in \Delta^{n}$.

La $k$-adgc, $G_{\sigma}=A_{\infty}\left(\mathbf{E}_{\sigma}\right)$ est munie d'une structure de $A_{\infty}(|\sigma|)$-module via $\sigma \circ p_{\sigma}$. Les opérateurs $\partial_{i}: \operatorname{Sing}\left(E_{\partial_{i} \sigma}\right) \rightarrow \operatorname{Sing}\left(E_{\sigma}\right)$ et $s_{j}: \operatorname{Sing}\left(E_{s_{j} \sigma}\right) \rightarrow \operatorname{Sing}\left(E_{\sigma}\right)$ induisent $\delta_{i}: \mathbf{E}_{\partial_{i} \sigma} \rightarrow \mathbf{E}_{\sigma}$ et $s_{j}: \mathbf{E}_{s_{j} \sigma} \rightarrow \mathbf{E}_{\sigma}$. On prend alors $\partial_{i}=A_{\infty}\left(\delta_{i}\right), s_{j}=$ $A_{\infty}\left(s_{j}\right)$ comme opérateurs pour le système $G$.

On construit à partir de $G$ un système $\widetilde{G} \operatorname{sur} \operatorname{Sing}(X)$ par

$$
\widetilde{G}_{\sigma}=\left(G_{\sigma} \otimes W, d_{\widetilde{\boldsymbol{\theta}}_{\sigma}}\right), \quad \partial_{i} \otimes \text { id }, \quad s_{j} \otimes \text { id. }
$$

Il reste à montrer que $\widetilde{G}$ est un système local. Pour chaque $\sigma: \Delta^{n} \rightarrow X$, on a un isomorphisme $H^{*}\left(\widetilde{G}_{\sigma}\right) \cong H_{\widetilde{\boldsymbol{\Theta}}_{\sigma}}^{*}\left(A_{\infty}\left(\mathbf{E}_{\sigma}\right) ; W\right)$; d'autre part, le Théorème 4.7 entraîne l'existence d'un système local $H$ sur $\operatorname{Sing}\left(\Delta^{n}\right)$ tel que

$$
H_{\widetilde{\boldsymbol{\Theta}}_{\sigma}}^{*}\left(A_{\infty}\left(\mathbf{E}_{\sigma}\right) ; W\right) \cong H^{*}\left(A_{\infty}\left(\mathbf{E}_{\sigma}\right) ; \gamma^{*} p_{\sigma}^{*} H\right) \text {. }
$$


Avec la contractilité de $\Delta^{n}$, on peut supposer que $\gamma^{*} p_{\sigma}^{*} H$ est un système constant. Puisque $G$ est un système local extensible [Ha, 19.27], $\widetilde{G}$ l'est aussi.

Il est clair que $\widetilde{G}(X)$ est isomorphe à $\left(G(X) \otimes W, d_{\widetilde{\mathbf{\Theta}}}\right)$. D'après [Ha, Chapitre 19], $G(X) \cong A_{\infty}(E)$ donc $\widetilde{G}(X) \cong\left(A_{\infty}(E) \otimes W, d_{\widetilde{\boldsymbol{\Theta}}}\right)$ avec ce qui précède.

Dans notre cas, $\Gamma$ fournit un morphisme entre les systèmes locaux extensibles $F, \widetilde{G}$. Il reste à montrer que $\Gamma_{\sigma}^{*}$ est un isomorphisme, pour tout $\sigma$. Remarquons qu'il suffit de le faire pour les 0 -simplexes mais alors, par construction de $\Gamma$, on obtient l'isomorphisme $H^{*}(K(\pi, n)) \otimes W \cong \Lambda\left(V^{*}\right) \otimes W$, ce qui achève la démonstration.

\section{EXEMPLE}

Terminons ce travail avec un exemple; nous allons déterminer un modèle minimal non nilpotent du tore hyperbolique $T_{A}^{3}$, où $A$ est une matrice appartenant à $\mathrm{SL}_{2}(\mathbf{Z})$ de trace plus grande que 2 [GS].

L'espace $T_{A}^{3}$ s'obtient comme quotient de $T^{2} \times \mathbf{R}$ par l'action $(x, y, t) \rightarrow$ $(A(x, y), t+1)$. Le tore hyperbolique apparaît donc comme espace total d'une fibration

$$
T^{2} \rightarrow T_{A}^{3} \stackrel{p}{\rightarrow} S^{1}
$$

où l'action de $\pi_{1}\left(S^{1}\right)=\mathbf{Z}$ sur $\pi_{1}\left(T^{2}\right)=\mathbf{Z} \oplus \mathbf{Z}$ est donnée par l'application $\chi: \mathbf{Z} \rightarrow \operatorname{Aut}_{\mathbf{Z}}(\mathbf{Z} \oplus \mathbf{Z}), \chi(1)=A$.

La matrice diagonalisable $A$ ayant toutes ses valeurs propres réelles et positives, $\chi$ est la restriction à $\mathbf{Z}$ d'une application, aussi notée $\chi$, de $\mathbf{R}$ dans $\operatorname{Aut}_{\mathbf{R}}(\mathbf{R} \oplus \mathbf{R})$, définie par $\chi(t)=A^{t}$. Ainsi, par le Corollaire 3.7, l'action $\chi$ provient d'un système algébrique $\left(\boldsymbol{\theta}, \mathbf{R}^{2}\right)$, où $\boldsymbol{\theta}=\alpha \otimes \log (\chi(1))$ avec $\alpha$ une forme volume de $S^{1}$.

Notons $\operatorname{Sing}^{\infty}\left(S^{1}\right)$ le sous-ensemble simplicial de $\operatorname{Sing}\left(S^{1}\right)$ formé des simlexes singuliers $C^{\infty}$. Le système local $A_{\infty}$ étant extensible, toute forme sur $\operatorname{Sing}^{\infty}\left(S^{1}\right)$ peut être étendue en une forme $\operatorname{sur} \operatorname{Sing}\left(S^{1}\right)$ [Ha, 12.21]. Soit $\gamma: \Omega\left(S^{1}\right) \rightarrow A_{\infty}\left(\right.$ Sing $\left.^{\infty}\left(S^{1}\right)\right)$ défini par $(\gamma \Phi)_{\sigma}=A_{\infty}(\sigma) \Phi[\mathrm{Ha}, 15.19]$; on choisit, et on note aussi $\alpha \in A_{\infty}\left(S^{1}\right)$, une extension de l'image de $\alpha$ par $\gamma$. L'action $\chi$ peut être vue comme induite par le système $\left(\boldsymbol{\Theta}, \mathbf{R}^{2}\right)$, considéré comme système sur $A_{\infty}\left(S^{1}\right)$.

D'autre part, remarquons que la classe caractéristique de la fibration $p$ est nulle. Le morphisme $\Gamma \mathrm{du}$ Théorème 5.1 s'écrit alors:

$$
\Gamma:\left(A_{\infty}\left(S^{1}\right) \otimes \Lambda(y, z), D\right) \rightarrow A_{\infty}\left(T_{A}^{3}\right)
$$

avec $|y|=1,|z|=1$,

$$
D y=\alpha \otimes y, \quad D z=-\alpha \otimes z .
$$

Rappelons que $\Gamma$ induit un isomorphisme en cohomologie pour tous les systèmes sur l'adgc $A_{\infty}\left(S^{1}\right)$. Dans [Go] sous des hypothèses raisonnables de connexité, on montre l'existence et l'unicité de tels modèles algébriques.

Si on ne s'intéresse qu'à la cohomologie réelle, on peut remplacer $A_{\infty}\left(S^{1}\right)$ par son modèle minimal $h: \Lambda(X) \rightarrow A_{\infty}\left(S^{1}\right), h(x)=\alpha$. On obtient alors une flèche

$$
\Gamma^{\prime}:(\Lambda(x, y, z), D) \rightarrow A_{\infty}\left(T_{A}^{3}\right)
$$

induisant un isomorphisme pour la cohomologie à coefficients dans $\mathbf{R}$. 


\section{RÉFÉRENCES}

[Ba] H. Baues, Obstruction theory, Lecture Notes in Math., vol. 628, Springer-Verlag, New York, 1977.

[DS] P. Deligne et D. Sullivan, Fibrés vectoriels complexes à groupe structural discret, C.R. Acad. Sci. Paris 281 (1975), 1081-1083.

[GS] E. Ghys et V. Sergiescu, Stabilité et conjugaison différentiable pour certains feuilletages, Topology 19 (1980), 179-197.

[Go] A. Gómez Tato, Modèles minimaux résolubles, J. Pure. Appl. Algebra (à paraître).

[GHV] W. Greub, S. Halperin, et R. Vanstone, Connections, curvature and cohomology, vol. II, Academic Press, 1972.

[Gr] P. Grivel, Formes différentielles et suites spectrales, Ann. Inst. Fourier (Grenoble) 29 (1979), 17-37.

[Ha] S. Halperin, Lectures on minimal models, Mém. Soc. Math. France (N.S.) 9-10 (1983).

[Hi] G. Hirsch, Sur la structure multiplicative de l'anneau de cohomologie d'un espace fibré, C. R. Acad. Sci. Paris 230 (1950), 46-49.

[Su] D. Sullivan, Infinitesimal computations in topology, Inst. Hautes Études Sci. Publ. Math. 47 (1977), 269-331.

Departamento de Xeometría e Topoloxía, Universidade de Santiago de Compostela, Colexio Universitario de Lugo, 27071 Lugo, Spain 